\title{
Flow cytometric assessment of myeloperoxidase in bovine blood neutrophils and monocytes
}

\author{
Elke Depreester, ${ }^{*}$ Evelyne Meyer, $\dagger^{1}$ Kristel Demeyere, $\dagger^{1}$ Mieke Van Eetvelde, ${ }^{*}$ Miel Hostens, ${ }^{*}$ \\ and Geert Opsomer*2 \\ *Department of Reproduction, Obstetrics and Herd Health, and \\ †Laboratory of Biochemistry, Faculty of Veterinary Medicine, Ghent University, Salisburylaan 133, Merelbeke 9820, Belgium
}

\begin{abstract}
Myeloperoxidase (MPO) is a lysosomal peroxidase enzyme mainly stored in the azurophilic granules of neutrophils playing an important role in innate immunity for first-line protection against microorganisms in many species including cattle. As such, determination of MPO has become of great interest for the diagnosis of infectious and inflammatory diseases in multiple species such as humans. In cattle, MPO determination is rarely done because methods to assess MPO in this species are limited: functional assays have been described earlier, but so far, the quantification of MPO at the single cell level has not been done yet. In the present paper, an innovative flow cytometric method to assess MPO in blood leukocytes of dairy cattle is described. A commercial anti-bovine MPO was used following density gradient separation to isolate polymorphonuclear (PMN) and mononuclear (MN) leukocytes from blood. Identification of PMN and MN, subdivided in monocytes and lymphocytes, was based on the expression of the surface markers CH138A and CD172A. The optimized protocol was subsequently evaluated on blood samples of 17 Holstein Friesian heifers. Myeloperoxidase expression was measured flow cytometrically and visualized by fluorescence microscopic imaging of sorted PMN and MN populations. We suggest this innovative method to be useful in the field for early detection of cows at higher risk for inflammatory diseases such as mastitis and metritis during the transition period.
\end{abstract}

Key words: dairy cow, myeloperoxidase, flow cytometry, blood leukocyte

\section{INTRODUCTION}

The inflammatory response basically aids in protecting against invasion of pathogens and is necessary for

Received October 20, 2016.

Accepted April 25, 2017.

${ }^{1}$ These authors contributed equally to the work.

${ }^{2}$ Corresponding author: geert.opsomer@ugent.be repair of tissue injury, but may give rise to deleterious consequences when deregulated. Dairy cattle with a high genetic merit for milk production are more prone to a variety of metabolic and infectious diseases (Drackley et al., 2001; Goff, 2006) during the transition from late pregnancy to early lactation. During that period, both the dairy cow's metabolism and immune system undergo drastic adaptations (Bertoni et al., 2009) supporting the switch from pregnancy to lactation. Numerous studies suggest that the massive lipid mobilization, associated with the negative energy balance during early lactation, compromises the cows' immune cell function. These dysfunctional host immune responses are regarded as the underlying cause for the increased incidence of transition disorders such as metritis and mastitis (Contreras et al., 2010; Sordillo and Raphael, 2013). Trevisi et al. (2012) summarized that parturition and the concomitant initiation of milk production in modern dairy cows are characterized by a (pro-)inflammatory state giving rise to an increased incidence of infectious and inflammatory diseases impairing both the welfare of the cows and the income of the farmer.

Myeloperoxidase (MPO) plays a key role in mammalian innate immunity. It is stored in the lysosomes of granulomonocytic immune cells and is secreted upon their activation (Borregaard and Cowland, 1997). The MPO enzyme transforms $\mathrm{H}_{2} \mathrm{O}_{2}$ into hypochlorous acid, the latter being highly efficient in killing pathogenic invaders such as bacteria, but also potentially causing cytotoxic damage to the host tissue. In human medicine, MPO has evolved toward an important inflammation biomarker and is used as a diagnostic tool for acute bacterial infections (Kulander et al., 2001) and recently also in the diagnosis of wound (Blokhuis-Arkes et al., 2015) and urinary tract infections (Karlsen and Dong, 2015).

In analogy with human medicine, MPO could be an interesting molecule for evaluating bovine immune function against bacterial infection. However, in marked contrast to human medicine, immuno-assay techniques for quantification of the MPO protein are rarely described in cattle. Functional assays, based on 
o-dianisidine, cellular chemiluminescence, or radioactive iodination assay, have been described by Zwahlen et al. (1992), Mehrzad et al. (2001), and Kampen et al. (2004), respectively. Of interest, the group of Cooray (1994) developed an ELISA for detection of MPO in bovine milk as a marker for mastitis more than 2 decades ago. In 1995, the same group described an adapted protocol (Cooray et al., 1995) using a doubleantibody ELISA to measure MPO in bovine serum and neutrophil extracts. Although such an ELISA can be applied for quantification of both intracellular as well as surface MPO, it cannot detect MPO at the single cell level as in flow cytometry (De Schauwer et al., 2012).

Therefore, the aim of the current research was to develop a single cell flow cytometric method for MPO quantification in innate immune cells isolated from bovine blood.

\section{MATERIALS AND METHODS}

\section{Isolation of Polymorphonuclear Granulocytic and Mononuclear Bovine Blood Cells}

Blood was collected by jugular venous puncture; 10 $\mathrm{mL}$ of blood was diluted 1:1 in Alsever's anticoagulant solution. The blood sampling technique was approved by the concerned Ethical Committee (EC 2013/124, Faculty of Veterinary Medicine, Ghent University). The $20 \mathrm{~mL}$ of diluted blood was layered on $10 \mathrm{~mL}$ of Lymphoprep density gradient medium $(1.077 \mathrm{~g} / \mathrm{mL}$; Stemcell Technologies, Cambridge, UK). After centrifugation $\left(900 \times g, 30 \mathrm{~min}, 20^{\circ} \mathrm{C}\right)$, mononuclear leukocytes (MN) were collected between the plasma and Lymphoprep layer and a red blood cell lysis step was needed to isolate the PMN from the lower layer. The lysis buffer consisted of $2.6 \mathrm{~g} / \mathrm{L}$ of Tris-aminomethane $\left(\mathrm{C}_{4} \mathrm{H}_{11} \mathrm{NO}_{3}\right)$ and $7.4 \mathrm{~g} / \mathrm{L}$ of ammonium chloride $\left(\mathrm{NH}_{4} \mathrm{Cl}\right)$ in water at $\mathrm{pH}$ 7.4. The cells were finally washed twice $(200 \times g$, $10 \mathrm{~min}, 4^{\circ} \mathrm{C}$ ) in PBS to remove remaining Lymphoprep. Isolated cells were resuspended in PBS $+1 \%$ BSA and counted on the FACSArray flow cytometer (Becton Dickinson and Company, Franklin Lakes, NJ) with Trucount beads (Becton Dickinson and Company). Before further labeling steps, a viability check was performed on the FACSCanto with propidium iodide (final concentration $1 \mu \mathrm{g} / \mathrm{mL}$ ).

\section{CH138 Immunolabelling of Bovine Blood PMN}

The primary anti-bovine granulocyte monoclonal antibody (CH138A) was used by Piepers et al. (2009) as an indirect fluorescent marker to identify milk neutrophils in bovine milk samples. In the present study, 2 individual samples were labeled with this surface marker before incubation with the anti-MPO antibody, as a confirmation of the discrimination of PMN (identified as CH138A positive), based on their forward-scatter (FS) and side-scatter (SS) characteristics. The PMN cells $\left(4 \times 10^{5}\right)$ were incubated with the primary mouse antibovine granulocyte monoclonal antibody (CH138A) at a final concentration of $10 \mu \mathrm{g} / \mathrm{mL}$ in RPMI $+1 \%$ BSA [reference CH138A (1 mg/mL), VMRD Inc., Pullman, WA] for $30 \mathrm{~min}$ at $37^{\circ} \mathrm{C}$ (Piepers et al., 2009). After incubation the cells were washed 2 times $(200 \times g, 10$ $\min , 20^{\circ} \mathrm{C}$ ). The incubation and wash medium consisted of RPMI $+1 \%$ BSA. Next, cells were incubated with a secondary antibody (goat anti-mouse IgM Alexa-647; A-21238, Molecular Probes, Invitrogen, Carlsbad, CA) for $30 \mathrm{~min}$ on ice in the dark at a final concentration of $4.8 \mu \mathrm{g} / \mathrm{mL}$ in RPMI $+1 \%$ BSA. Cells were washed 3 times before the MPO labeling.

\section{CD172A Immunolabelling of Bovine Blood Mononuclear Cells}

CD172A is a surface protein that is expressed on monocytes and macrophages (Pilling et al., 2009; Hussen et al., 2013). Two individual samples were labeled with this surface marker before incubation with the anti-MPO antibody, as a confirmation of the discrimination of monocytes (identified as CD172A positive) and lymphocytes (CD172A negative) based on their FS and SS characteristics. The mononuclear leukocytes (4 $\times 10^{5}$ ) were incubated for $30 \mathrm{~min}$ in a dark room at 2 to $8^{\circ} \mathrm{C}$ with a primary monoclonal PerCP-Cy5 fluorescently labeled anti-CD172A mouse-antibody (MCA2041C, Bio-Rad, Hercules, CA) or isotype-matched antibody (MCA691C, Bio-Rad) as negative control, at a final concentration of $2 \mu \mathrm{g} / \mathrm{mL}$ (as advised by Hussen et al., 2013). After incubation, the cells were washed once $\left(200 \times g, 10 \mathrm{~min}, 20^{\circ} \mathrm{C}\right)$. The incubation and wash medium used in these and the following steps consisted of PBS $+1 \%$ BSA.

\section{MPO Immunolabelling-Titration Curves of Polymorphonuclear Granulocytic and Mononuclear Bovine Blood Cells}

Both PMN and MN were first fixed for $15 \mathrm{~min}$ in reagent A of Fix\&Perm (Thermo Fisher Scientific, Waltham, MA) at room temperature in the dark. After washing $\left(500 \times g, 5 \mathrm{~min}, 4^{\circ} \mathrm{C}\right)$, cells were permeabilized for 15 min in the dark in Fix\&Perm (reagent B) and then washed again. To minimize aspecific binding, a blocking step was performed by incubation with $20 \%$ goat serum in incubation medium for 30 min before antibody staining. After one wash step, labeling was done indirectly with a rabbit anti-bovine MPO polyclonal 


\section{A)}

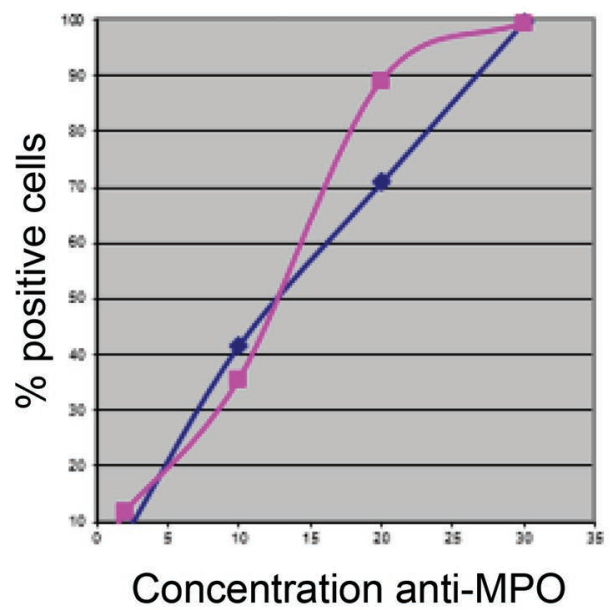

B)

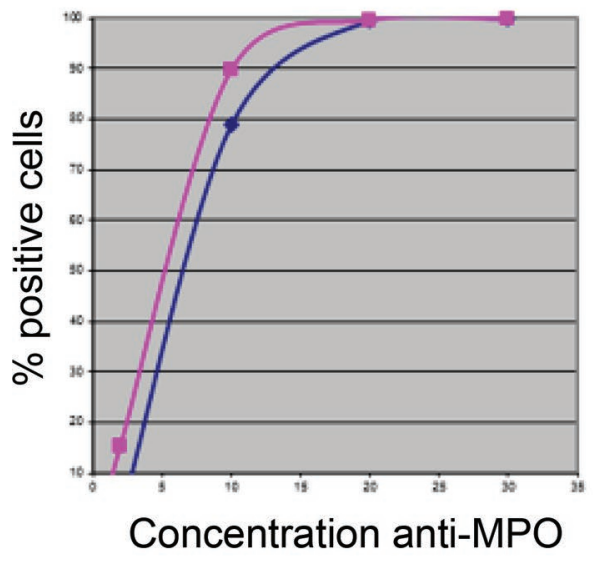

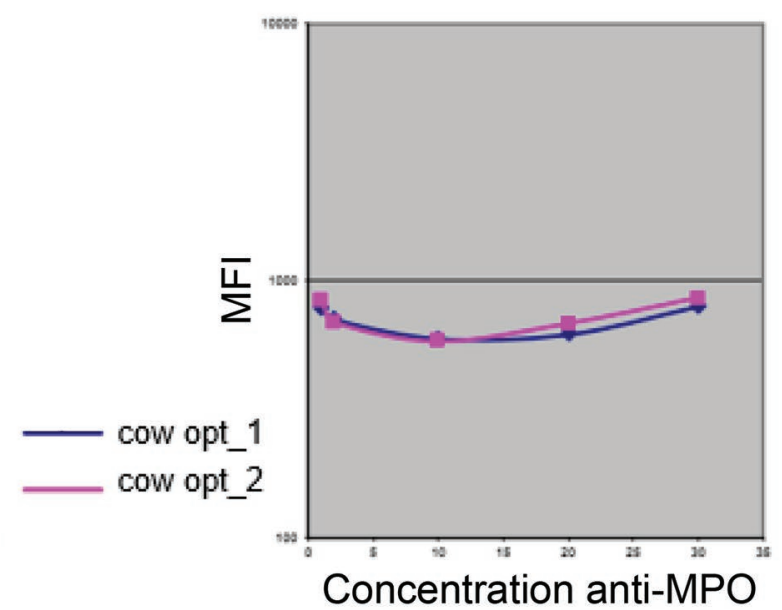

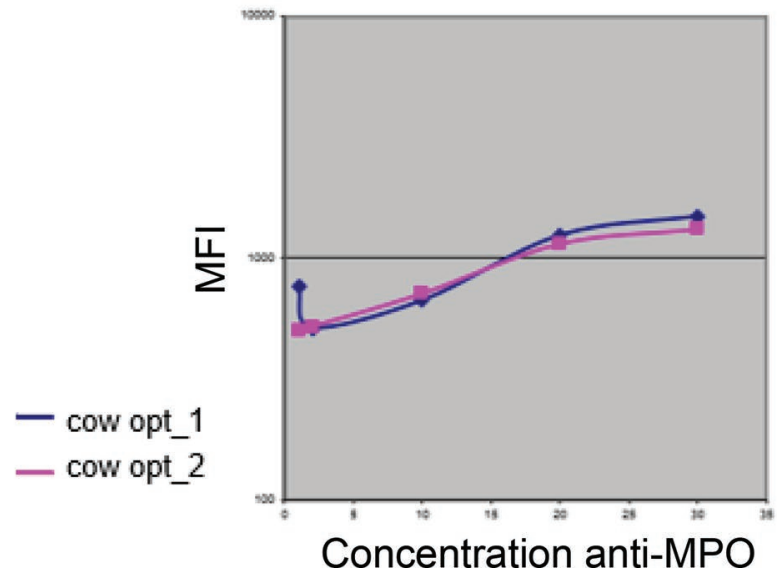

Figure 1. (A) Titration curve of the anti-myeloperoxidase (MPO) primary antibody in PMN: percentage of MPO-positive PMN (left) and mean fluorescence intensity (MFI; right) at different test concentrations $(n=2)$. (B) Titration curve of the anti-MPO antibody in mononuclear cells (MN): percentage of MPO-positive cells (left) and MFI (right) at different test concentrations $(\mathrm{n}=2)$. The optimization of the protocol was done on 2 cows (opt_1 and opt_2). Color version available online.

antibody (Antibodies online, ABIN737076, Aachen, Germany) for $30 \mathrm{~min}$ at room temperature. To choose the optimal concentration, a titration curve was made for 2 individual cows and 5 different concentrations (1, $2,10,20$, and $30 \mu \mathrm{g} / \mathrm{mL})$. Based on these titration curves (Figure 1A and 1B), a final concentration of 30 and $20 \mu \mathrm{g} / \mathrm{mL}$ in PMN and MN cells, respectively, was used. As a negative isotype control, a rabbit IgG (at the same concentration as the primary antibody) was used that was purified from rabbit serum by a protein An affinity chromatography (kind gift from E. Cox, Department of Virology, Parasitology and Immunology, Faculty of Veterinary Medicine, Ghent University). After washing, the cells were incubated with a secondary antibody, a goat anti-rabbit IgG Alexa 488 (Invitrogen, A11070) at a final concentration of $2 \mu \mathrm{g} / \mathrm{mL}$ for $30 \mathrm{~min}$ at room temperature. Finally, the MPO expression of
PMN and MN cells was measured on a FACSCanto flow cytometer (Becton Dickinson and Company). After gating, a minimum of $1 \times 10^{4}$ cells were analyzed.

\section{Flow Cytometry and Fixation}

Flow cytometric measurements were performed immediately after staining on a FACSCanto flow cytometer (Becton Dickinson and Company, Erembodegem, Belgium), with $488 \mathrm{~nm}$ excitation from a blue solidstate laser at $50 \mathrm{~mW}$. Optical filters were set up such that CD172 PerCp-Cy5 was measured above $670 \mathrm{~nm}$ (FL3) and MPO fluorescein isothiocyanate (FITC) was measured at $530 \pm 15 \mathrm{~nm}$ (FL1). All acquired data were processed using FACSDiva software (Becton Dickinson and Company, Franklin Lakes, NJ). 
For weekly validation of the flow cytometer, Rainbow Calibration Particles (8 peaks, catalog no. 559123, Becton Dickinson and Company) were used, every rainbow particle containing a mixture of 8 fluorophores that are excited at wavelengths from 365 until $650 \mathrm{~nm}$. The rainbow particles have emission spectra compatible with many common fluorophores used for immunofluorescent staining with flow cytometric analysis. The mean fluorescence intensity (MFI) was compared with the MFI at the previous measurement; if the deviation was higher than $1.5 \%$, a correction of the voltage was made.

\section{Sorting and Fluorescence Microscopy}

As an additional control, PMN, monocytes, and lymphocytes were sorted on a FACSAria III (Becton Dickinson and Company). The PMN were identified based on the FS/SS characteristics, as described earlier. Within the MN, CD172A-positive monocytes and CD172A-negative lymphocyte subpopulations were sorted separately. After nuclear staining with Hoechst, sorted cell populations were visualized and characterized by fluorescence microscopy (Leica DMRBE 301-877.011, Leica Microsystems, Wetzlar, Germany).

\section{Proof of Concept and Statistical Analysis}

As a proof-of-concept study, the optimized flow cytometric MPO protocol was subsequently evaluated on blood samples of 17 Holstein Friesian heifers, 8 of them between 14 and $5 \mathrm{~d}$ before the expected calving date and 9 of them between 5 and $14 \mathrm{~d}$ after parturition.
All data were analyzed using the dedicated FACSDiva software (Becton Dickinson and Company) and subsequently exported to Excel (Excel 2013, Microsoft, Redmond, WA). SPSS Statistics version 24 (IBM Corp., Armonk, NY) was used to calculate descriptive statistic parameters and perform the nonparametrical Mann Whitney U-test.

\section{RESULTS}

\section{Optimization of the Immunolabelling Protocol}

Titration Curves. A titration of the primary MPO antibody was performed for which both the percentage of positive cells (\%) and the MFI of cells from 2 individual cows were measured at increasing concentrations of the anti-MPO polyclonal antibody. The titration curves obtained for these 2 parameters in the PMN and MN cell populations are shown in Figure 1A and 1B. First, it was evaluated at which concentration $100 \%$ of the cells were MPO positive. Once this saturation was reached, the MFI had to be stable. In PMN, both criteria were satisfied at a final concentration of $30 \mu \mathrm{g} /$ $\mathrm{mL}$. In MN, at a concentration of $20 \mu \mathrm{g} / \mathrm{mL}$, the MFI was not yet maximal. Still, as saturation was reached at that point and incubation time, reaction volume, and incubation circumstances were kept constant, a final concentration of $20 \mu \mathrm{g} / \mathrm{mL}$ was chosen in $\mathrm{MN}$.

Gating Strategy. To gate the single cells, both in PMN and MN, 3 consecutive steps were used: a primary gate was placed on the FS cytogram area (FSC-A) versus width (FSC-W) signal dot-plot, this population was then displayed as the SS cytogram area (SSC-A)
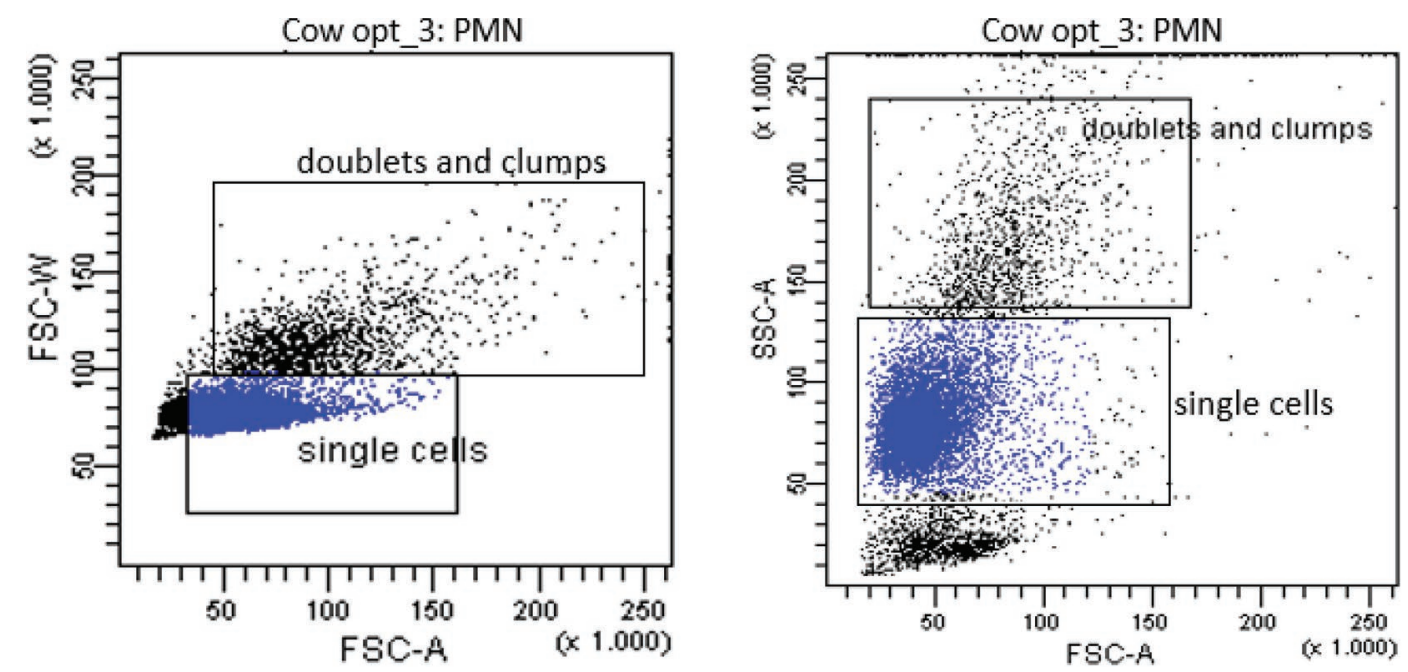

Figure 2. Gating strategy for single cells, illustrated in PMN cell population: forward-scatter cytogram area (FSC-A) versus forward-scatter cytogram width (FSC-W) signal dot-plot (left). The PMN cells on a side-scatter cytogram area (SSC-A) versus forward-scatter cytogram area (FSC-A) signal dot-plot (right). This gating strategy was optimized on cow opt_3. 
versus width (SSC-W) dot-plot to discriminate for doublets and clumps, and finally backgated on a SSC-A versus FSC-A signal dot-plot to further exclude debris (Figure 2).

Within the single cell population in PMN cells, neutrophils were determined based on their FS/SS characteristics. This PMN gating was confirmed in 2 individual cows by performing an extra labeling with a primary anti-bovine granulocyte monoclonal antibody (CH138A) as described by Piepers et al. (2009) (Figure $3 \mathrm{~A})$.

Within the single cell gate in MN, 2 subpopulations could be discriminated based on their FS/SS characteristics with the monocytes having a higher SSC-A and
FSC-A signal than the lymphocytes. This was again confirmed in 2 individual cows by extra labeling with CD172A as a monocyte marker and backgating on

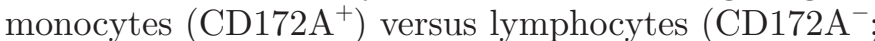
Figure 3B).

\section{MPO Expression in Bovine Blood Leukocytes}

Flow Cytometric Assessment of MPO. The MFI of the FITC signal was measured as parameter for the comparative MPO expression in the 3 blood leukocyte populations (PMN versus $\mathrm{MN}$, the latter consisting of monocytes and lymphocytes). All data were corrected for autofluorescence as well as for aspecific binding us-

A)
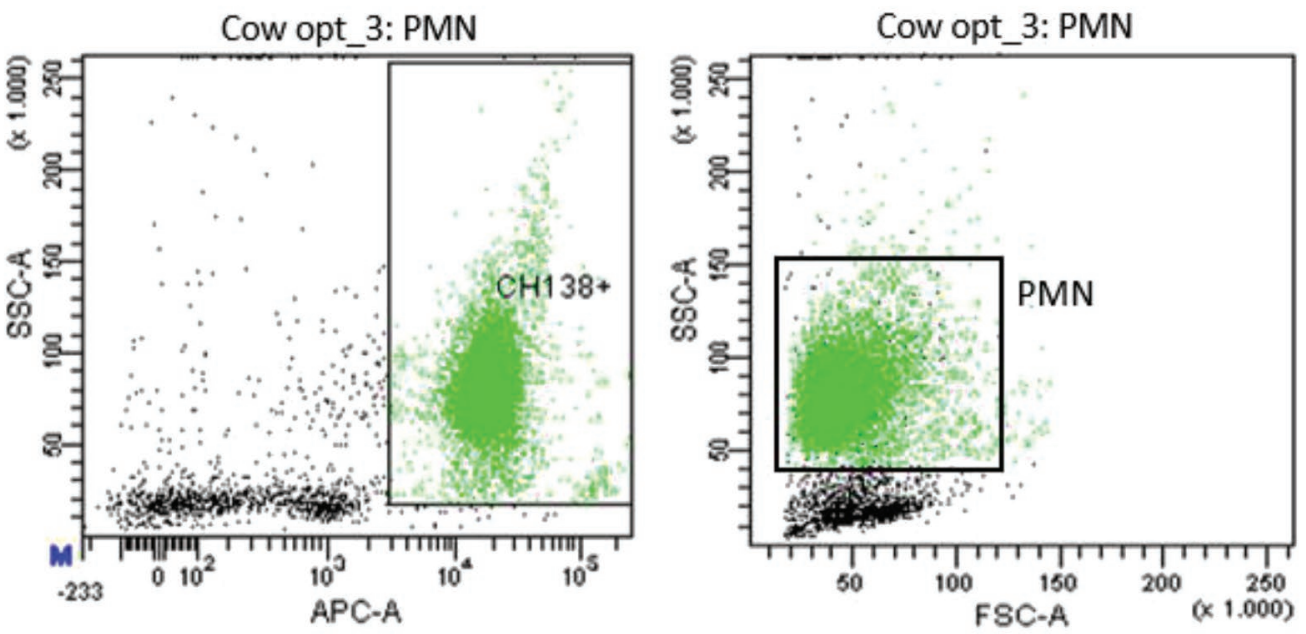

B)
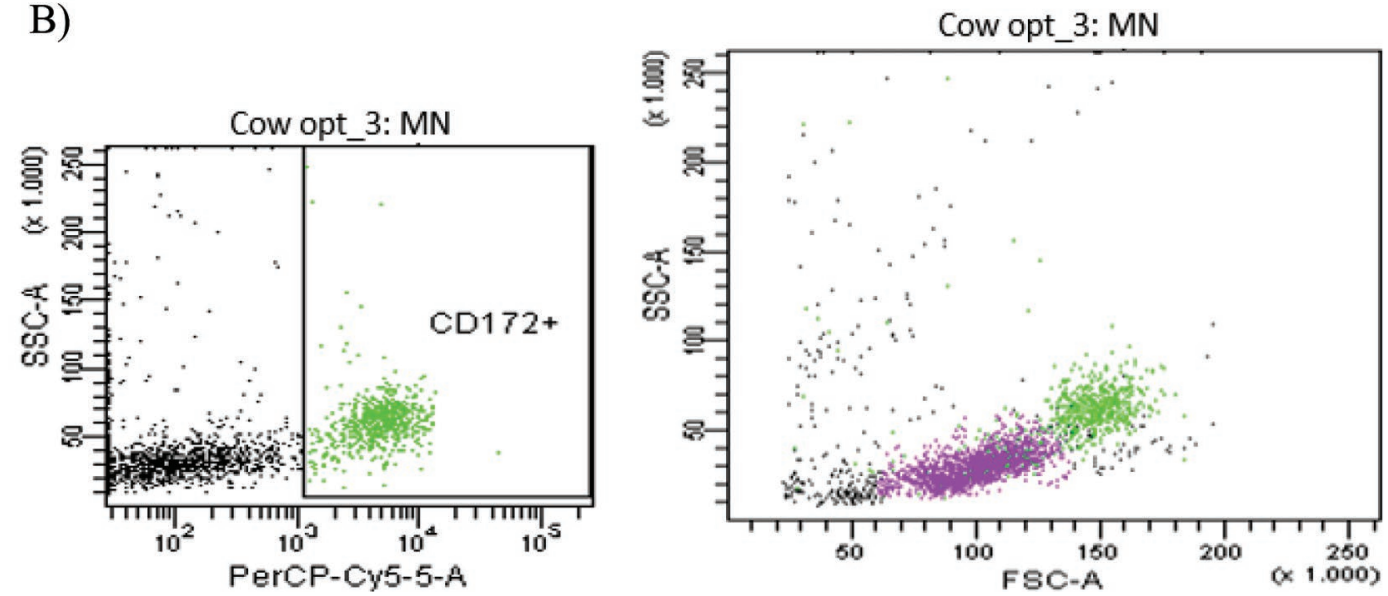

Figure 3. (A) Positivity for the PMN marker CH138A in the side-scatter cytogram area (SSC-A) versus allophycocyanin-area (APC-A) dot-plot (left) confirmed the forward-scatter cytogram area (FSC-A) versus SSC-A dot-plot identification of PMN after backgating of this CH138A-positive subpopulation as the cells with highest side-scatter characteristics (right). (B) Positivity for CD172A in the side-scatter cytogram area (SSC-A) versus PerCP Cy5-A (Bio-Rad, Hercules, CA) dot-plot (left) confirmed the forward-scatter cytogram area (FSC-A) versus SSC-A dot-plot identification of monocytes after backgating of this CD172A-positive subpopulation as the cells with highest side-scatter and forward-scatter characteristics (right). The CD172A-negative lymphocytes (purple) are confirmed to be the mononuclear leukocytes (MN) with the lowest forward-scatter and side-scatter characteristics. Results of the optimization are shown for cow opt_3. 
ing either isotype-matched or secondary $\mathrm{Ab}$ alone as negative controls (Figure 4A and 4B). All isotypes were matched to the immunoglobulin subtype, conjugated to the same fluorochrome, and used at the same fluorescence/protein concentration as the corresponding primary anti-MPO Ab.

In Figure 4B, the threshold for MPO positivity in the monocytes $\left(\mathrm{CD} 172 \mathrm{~A}^{+}\right)$is shown. However, the MFI of MPO in the MN cells showed a distribution with a low and a high peak. Backgating on FS/SS and CD172A expression identified the monocytes $\left(\mathrm{CD} 172 \mathrm{~A}^{+}\right.$, higher FS/SS) as the population with a higher MPO expres-

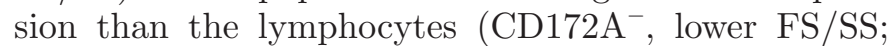
Figure 5).

Fluorescence Microscopic Assessment of MPO Expression After Sorting. Sorting, followed by fluorescence microscopy, confirmed the correct flow cytometric identification of the blood leukocyte sub- populations and their MPO content (Figure 6A-6C). Isolation of PMN was based on the high FS and SS signal of this population, as shown in Figure 2. For identification of monocytes and lymphocytes within the MN cell population, both FS/SS characteristics and CD172A expression were used, as described in Figure 3B (right). Microscopic identification of the sorted cell populations was based on the typical morphology of the nuclei and the surface and intracellular MPO expression was clearly visible.

\section{Proof of Concept of the Novel Flow Cytometric Method}

As a proof of concept, the optimized flow cytometric MPO protocol was subsequently evaluated on blood samples of 17 randomly chosen Holstein Friesian heifers, 8 of them between 14 and 5 d before the expected
A)

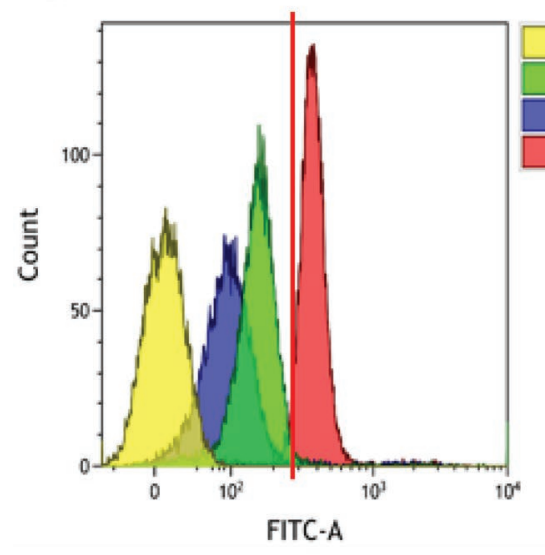

B)

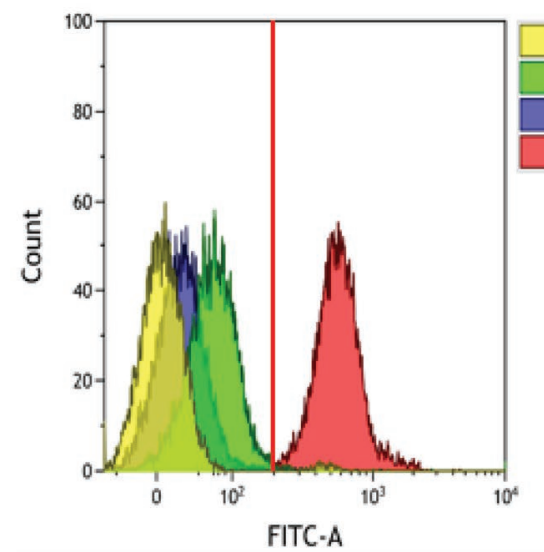

Cow oot 3 PMN: autofluorescence

Cow opt_3 PMN: isotype control

Cow opt_3 PMN: secondary

Cow opt_3 PMN: anti-MPO antibody and secondary antibody
Cow opt_3 MN: autofluorescence Cow opt_3 MN: isotype control Cow opt_3 MN: secondary antibody

Cow opt_3 MN: anti-MPO antibody and secondary antibody

Figure 4. (A) Mean fluorescence intensity of the fluorescein isothiocyanate (FITC) signal in the PMN with autofluorescence (yellow), isotype (green), only secondary (blue), and primary anti-myeloperoxidase (MPO) with secondary antibodies (red). The red line marks the chosen threshold for MPO positivity. (B) Mean fluorescence intensity of the FITC signal gated in the mononuclear leukocytes (MN) as shown in green in Figure 3B, with autofluorescence (yellow), isotype (green), only secondary (blue), and primary anti-myeloperoxidase (MPO) with secondary antibodies (red). The red line marks the chosen threshold for MPO positivity. Results of the optimization are shown for cow opt_3. 
calving date and 9 of them between 5 and $14 \mathrm{~d}$ after parturition. Before performing further flow cytometric analyses, concentrations of nonesterified fatty acids and BHB were determined (Supplemental Table S1; https:/ / doi.org/10.3168/jds.2016-12186). Every 6 wk, the individual SCC was measured as part of the official milk recording on the farm. The first SCC following parturition was taken into account: if higher than 150,000, the animal was excluded from further analysis. Individual metabolic parameters are shown in Supplemental Table S1 (https://doi.org/10.3168/jds.2016-12186). In none of the animals the threshold for subclinical ketonemia (fatty acid prepartum: $0.4 \mathrm{mmol} / \mathrm{L}$; BHB postpartum: $1.4 \mathrm{mmol} / \mathrm{L}$ ) was exceeded. The following analyses are preliminary data intended mainly to validate the novel flow cytometric methodology, yet allowing estimation of the expression of MPO in bovine PMN and monocytes as a basis for further research. The individual cow leukocyte population viabilities and differentiation are listed in Supplemental Table S2 (https://doi.org/ jds.2016-12186). The viability of the isolated blood leukocytes based on their propidium iodide negativity was on average $95.4 \%$ in PMN and $94.9 \%$ in MN ( $\mathrm{n}=$ 17). The distribution of the MPO expression in PMN and monocytes is shown in Figure 7 , with respective mean and SD of the MFI values for both populations. For the MN fraction, additional differentiation between monocytes and lymphocytes was done. Next to an intermediate expression in monocytes, a lower but unexpected expression of MPO was also found in lymphocytes (data not shown). Differences in MPO expression between PMN and MN populations were compared nonparametrically (Mann Whitney U-test), but did not turn out to be significant.

\section{DISCUSSION}

In the present study, we provide a new possibility for evaluating the cellular innate immunity in cows, complementing both the cytokine profile analysis (Plessers et al., 2015) and the viability assessment of bovine PMN with flow cytometry (Piepers et al., 2009). In further studies, we will examine whether our validated method can be used to more deeply research innate immunity of dairy cows in the transition period, for example with regard to fat cow syndrome.

Traditional flow cytometrical identification of isolated bovine blood leukocytes is based on their size and granularity as reflected in the FS/SS characteristics (Jain et al., 1991; Liu et al., 1993). In a first step, density gradient separation of PMN and MN and subsequent flow cytometrical analysis of these isolated cells revealed on the one hand a distinct high FS/SS population of PMN and on the other hand the MN population consisting of 2 subpopulations, the monocytes with intermediate FS/SS and the lymphocytes with low FS/SS characteristics. These observations on unlabelled innate immune cells confirm earlier reports by Jain et al. (1991) and Liu et al. (1993) on bovine blood leukocytes.

In a second step, this size- and granularity-based identification of PMN and MN was confirmed by labeling with the respective cell-specific surface markers CH138A and CD172A. The anti-bovine granulocyte
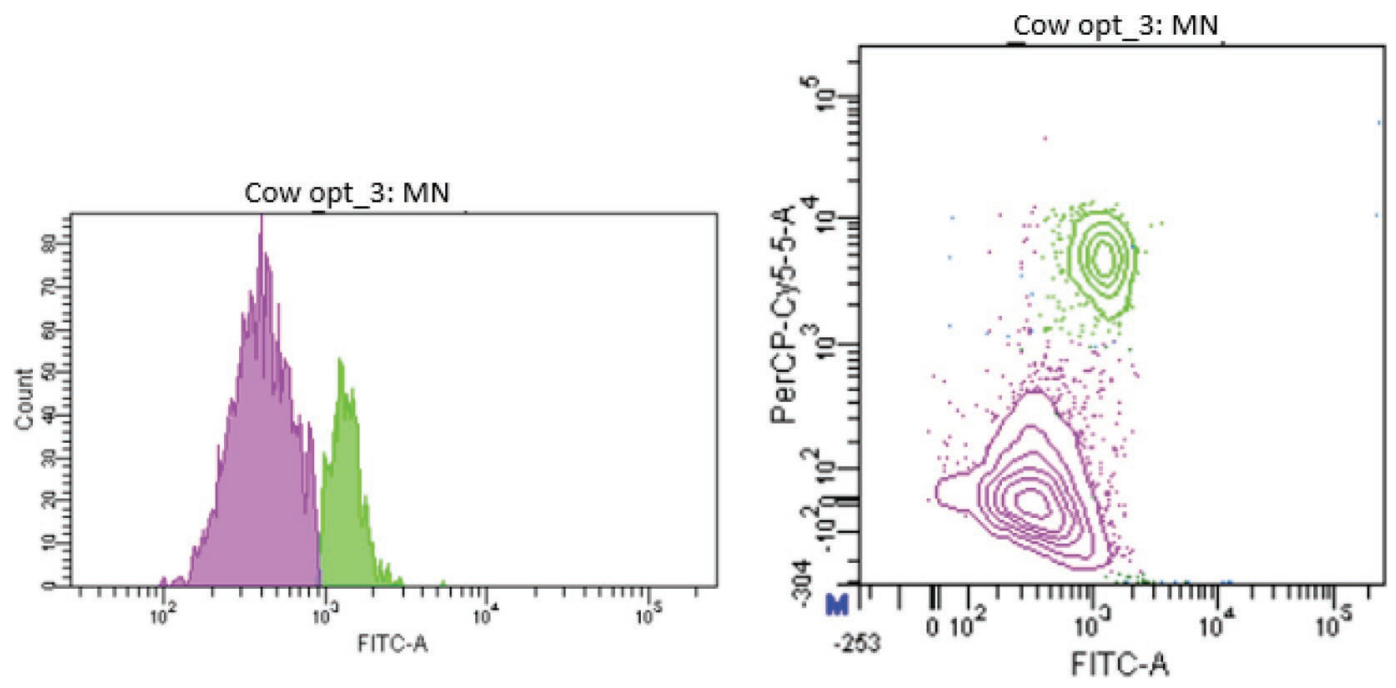

Figure 5. Fluorescein isothiocyanate (FITC)-A mean fluorescence intensity within the CD172A-labeled mononuclear leukocytes (MN; left) and contour plot with myeloperoxidase (MPO; FITC, x-axis) and CD172A (PerCP-Cy5, Bio-Rad, Hercules, CA; right). The CD172A-positive cells are colored green and CD172A-negative cells are shown in purple. The monocytes (CD172A subpopulation, green) have a higher MPO expression than the lymphocytes (CD172A ${ }^{-}$, purple). Results of the optimization are shown for cow opt_3. 
A)
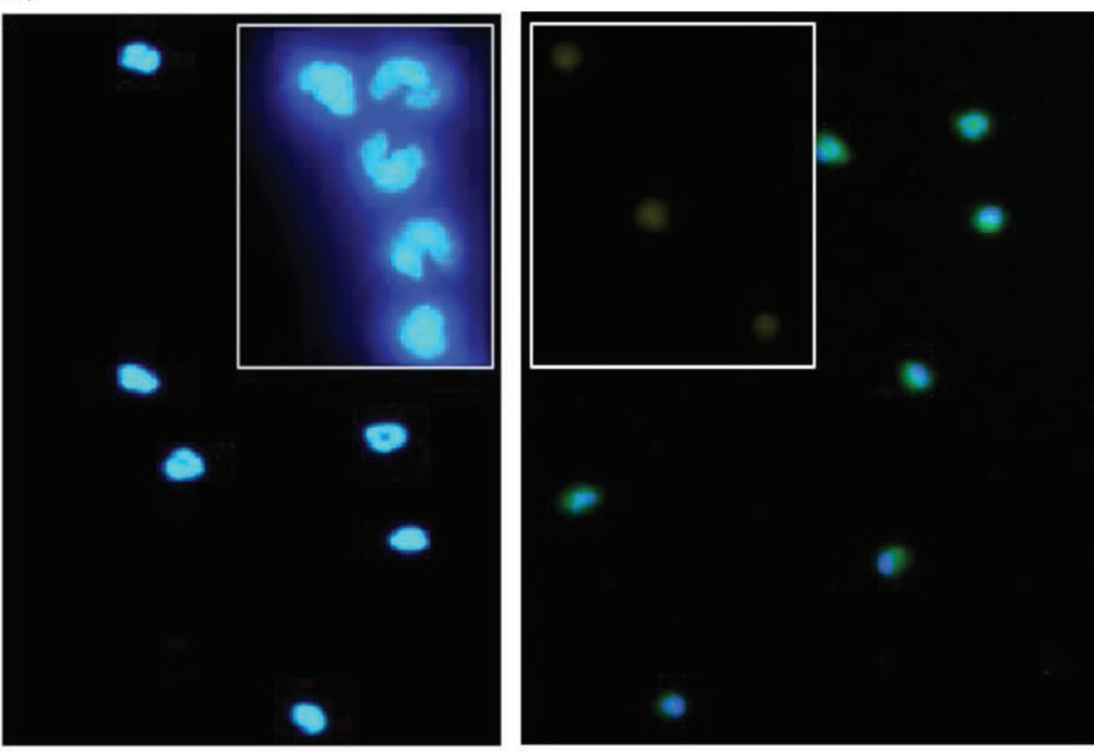

B)

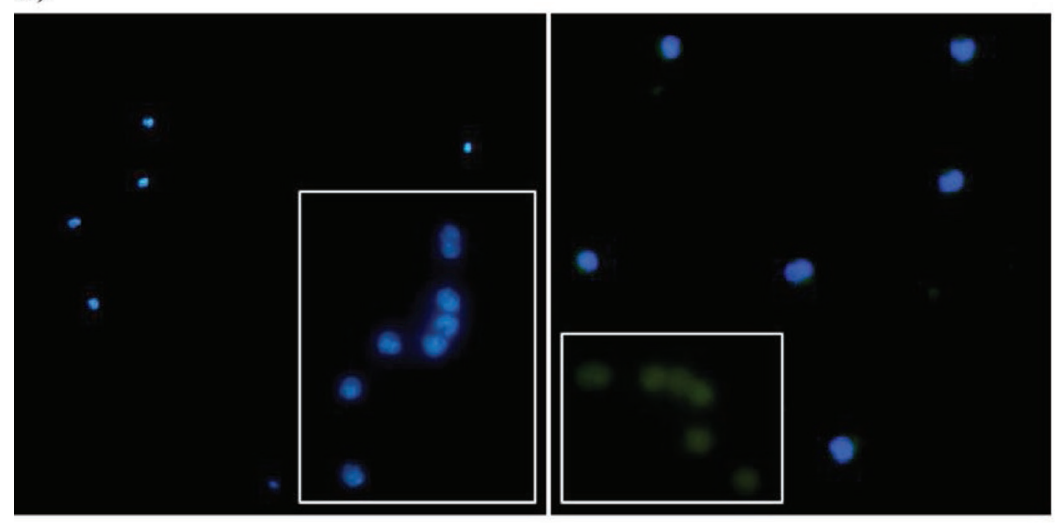

C)

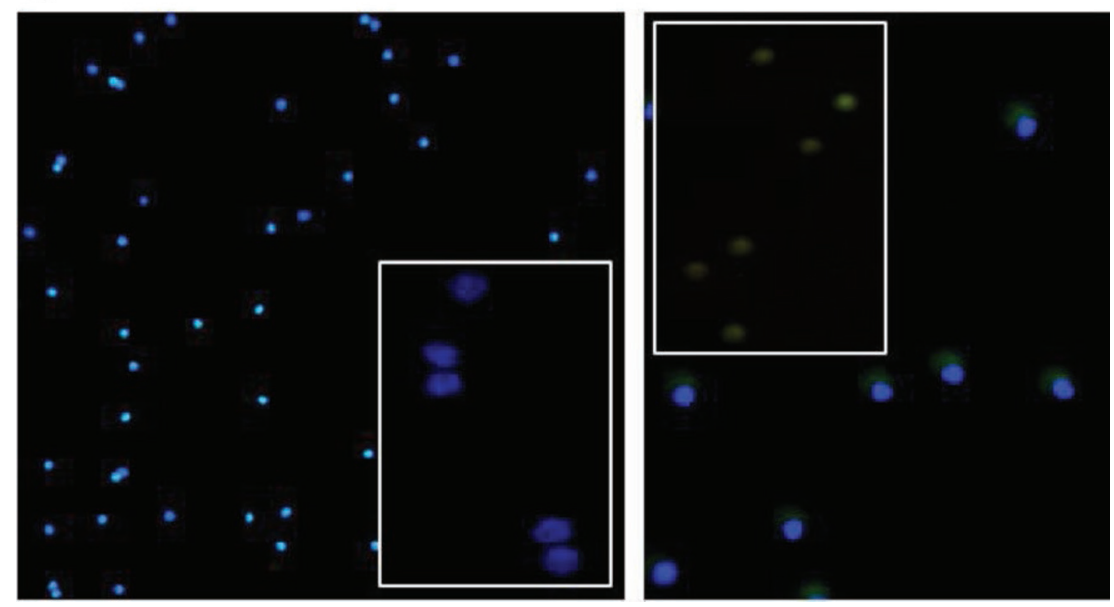

Figure 6. (A) Fluorescence microscopic images of PMN. Left: characteristic nuclear morphology after staining with Hoechst $(40 \times$; insert $100 \times$ ). Right: Myeloperoxidase (MPO) positivity after additional intracellular staining with fluorescein isothiocyanate (FITC, 40×, insert shows single staining for MPO). (B) Fluorescence microscopic images of monocytes. Left: characteristic nuclear morphology after staining with Hoechst $(40 \times$; insert $100 \times)$. Right: MPO positivity after additional intracellular staining with FITC (40×, insert shows single staining for MPO). (C) Fluorescence microscopic images of lymphocytes. Left: characteristic nuclear morphology after staining with Hoechst (40×; insert 100×). Right: MPO positivity after additional intracellular staining with FITC $(40 \times$, insert shows single staining for MPO). 


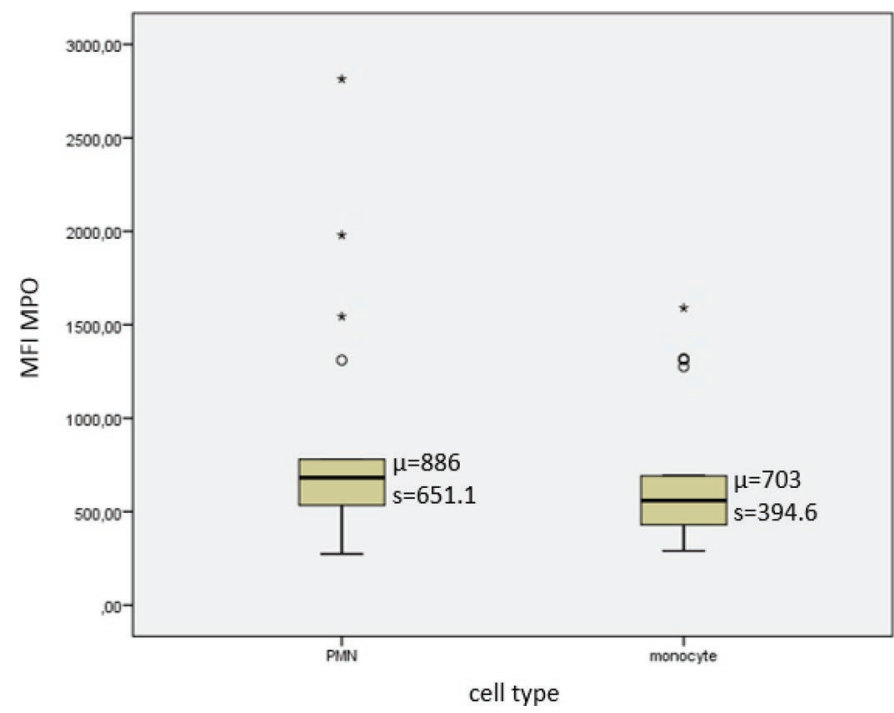

Figure 7. Box-whisker plot with the distribution of the myeloperoxidase (MPO) expression shown as mean fluorescence intensity (MFI) values in PMN and mononuclear leukocytes. Mean $(\mu)$ and SD (s) are given next to the respective boxplot. Outliers (outside the upper quartile) are marked as ${ }^{\circ}$ or ${ }^{*}$, being lower or higher, respectively, than 1.5 $x$ the interquartile range.

marker CH138A protocol was previously optimized by our group (Piepers et al., 2009) to differentiate bovine milk PMN from other somatic cells, whereas CD172A is a surface protein that was initially reported to be expressed on human monocytes and macrophages (Pilling et al., 2009). More recently, Hussen et al. (2013) described its suitability as a surface marker for monocytic leukocytes in bovine blood as well. Recently, Pomeroy et al. (2016) independently confirmed the elegant use of CD172a to gate all monocytes in bovine blood. Therefore, CD172a rather than the previously reported CD14 (Piepers et al., 2009) antibody was used in the present study.

In a third step, this surface marker identification of the bovine blood leukocyte populations was confirmed by fluorescence microscopic imaging of the respectively sorted cell populations. The nuclear morphology after staining with Hoechst was in accordance with that of the bovine white blood cell populations described by Adewoyin and Nwogoh (2014).

Following this stepwise optimization procedure, the cellular MPO expression was evaluated by labeling with a rabbit primary polyclonal antibody that had previously been successfully tested for immunohistochemical and immunofluorescence applications on murine cells (Li et al., 2013). Moreover, the reactivity against bovine MPO was tested and proof of specificity by Western blot analysis was delivered by the manufacturer (personal communication, data not shown). In PMN and MN, this anti-MPO antibody was used at a concentration of 30 and $20 \mu \mathrm{g} / \mathrm{mL}$, respectively, based on the establishment of saturation of the percentage of positive cells and stable MFI at that point. In compliance with the good practices of flow cytometry, corrections were made for autofluorescence as well as fluorescence of the corresponding $\operatorname{IgG}$ isotype and the secondary antibody before measuring the MPO. Moreover, as Rainbow calibration particles were used to correct for inter-analysis variation, the absolute MFI values could be reported as a reliable parameter for expression of MPO in PMN and MN.

Based on our data, we furthermore suggest that the 2-peak distribution of the MPO expression in the MN population can be used as an additional parameter to distinguish monocytes with a higher MPO-fluorescence from the lymphocytes with a lower MPO signal (Tay et al., 1998). This option was also given by our group for the differentiation of equine leukocytes (Wauters, 2012).

Following optimization of the novel flow cytometric protocol, the validity of this new method was illustrated by assessing the MPO expression in bovine leukocytes of 17 randomly chosen periparturient Holstein Friesian heifers. Although descriptive statistic parameters showed a high biological variability between individual animals, a general nonsignificant trend was observed with a higher expression of MPO in PMN than in monocytes in this proof-of-concept data set. Within the MN cell population, the lymphocytes had a relevant expression of MPO too. These findings are the first to be reported for the bovine species and correlate well with those of Tay et al. (1998), who described the flow cytometric discrimination of human PMN versus MN, subdivided in monocytes and lymphocytes based on their MPO expression. Of relevance, Okada et al. (2016) recently confirmed the production of MPO in human peripheral blood lymphocytes as well. Next, studies including a higher number of animals should be done to confirm this finding and to accurately quantify the differential expression of MPO in the different leukocyte populations isolated from bovine blood.

In conclusion, the results of the present technical note and proof-of-concept study provide a new method for quantification of the total MPO expression at the single cell level in PMN and MN of dairy cattle. We suggest that discrimination of total MPO levels in the quantification of surface and intracellular MPO might be performed in the future as an indicator of immune cell activation. Because MPO plays an important role in innate immunity and is a well-known innate immunity biomarker in human medicine, our novel protocol might be a promising step in further research about the role of innate immunity in transition disorders such as mastitis and metritis in high yielding dairy cows. 


\section{ACKNOWLEDGMENTS}

This research would not have been possible without the funding of the FWO (Fonds voor Wetenschappelijk Onderzoek Vlaanderen, Brussels, Belgium) PhD fellowship. Also the help of the technicians on Ghent University experimental herd and ILVO, and B. Weytens of Axelwalle, for taking the samples was greatly appreciated by the authors.

\section{REFERENCES}

Adewoyin, A., and B. Nwogoh. 2014. Peripheral blood film-A review. Ann. Ib. Postgrad. Med. 12:71-79.

Bertoni, G., E. Trevisi, and R. Lombardelli. 2009. Some new aspects of nutrition, health conditions and fertility of intensively reared dairy cows. Ital. J. Anim. Sci. 8:491-518.

Blokhuis-Arkes, M. H. E., M. Haalboom, J. van der Palen, A. Heinzle, E. Sigl, G. Guebitz, and R. Beuk. 2015. Rapid enzyme analysis as a diagnostic tool for wound infection: Comparison between clinical judgment, microbiological analysis, and enzyme analysis. Wound Repair Regen. 23:345-352.

Borregaard, N., and J. B. Cowland. 1997. Granules of the human neutrophilic polymorphonuclear leukocyte. Blood 89:3503-3521.

Contreras, G. A., N. J. O'Boyle, T. H. Herdt, and L. M. Sordillo. 2010. Lipomobilization in periparturient dairy cows influences the composition of plasma nonesterified fatty acids and leukocyte phospholipid fatty acids. J. Dairy Sci. 93:2508-2516.

Cooray, R. 1994. Use of bovine myeloperoxidase as an indicator of mastitis in dairy cattle. Vet. Microbiol. 42:317-326.

Cooray, R., C. G. B. Petersson, L. Moberg, and R. Bage. 1995. A sensitive double-antibody enzyme-linked immunosorbant assay for bovine myeloperoxidase and its application to serum and neutrophil extracts. Zentralbl. Veterinarmed. B 42:481-491.

De Schauwer, C., S. Piepers, G. R. Van de Walle, K. Demeyere, M. K. Hoogewijs, J. L. J. Govaere, K. Braeckmans, A. Van Soom, and E. Meyer. 2012. In search for cross-reactivity to immunophenotype equine mesenchymal stromal cells by multicolor flow cytometry. Cytometry A 81:312-323.

Drackley, J. K., T. R. Overton, and G. N. Douglas. 2001. Adaptations of glucose and long-chain fatty acid metabolism in liver of dairy cows during the periparturient period. J. Dairy Sci. 84(Suppl.):E100-E112.

Goff, J. P. 2006. Major advances in our understanding of nutritional influences on bovine health. J. Dairy Sci. 89:1292-1301.

Hussen, J., A. Duevel, O. Sandra, D. Smith, I. M. Sheldon, P. Zieger, and H.-J. Schuberth. 2013. Phenotypic and functional heterogeneity of bovine blood monocytes. PLoS One 8:e71502.

Jain, N. C., M. J. Paape, and R. H. Miller. 1991. Use of flow-cytometry for determination of differential leukocyte counts in bovine blood. Am. J. Vet. Res. 52:630-636.

Kampen, A. H., T. Tollersrud, S. Larsen, J. A. Roth, D. E. Frank, and A. Lund. 2004. Repeatability of flow cytometric and classical measurement of phagocytosis and respiratory burst in bovine polymorphonuclear leukocytes. Vet. Immunol. Immunopathol. 97:105-114.

Karlsen, H., and T. Dong. 2015. Biomarkers of urinary tract infections: State of the art, and promising applications for rapid strip-based chemical sensors. Anal. Methods 7:7961-7975.
Kulander, L., K. Pauksens, and P. Venge. 2001. Soluble adhesion molecules, cytokines and cellular markers in serum in patients with acute infections. Scand. J. Infect. Dis. 33:290-300.

Li, Y. Y., B. Yuece, H. M. Cao, H. X. Lin, S. Lv, J. C. Chen, S. Ochs, A. Sibaev, E. Deindl, C. Schaefer, and M. Storr. 2013. Inhibition of p38/Mk2 signaling pathway improves the anti-inflammatory effect of WIN55 on mouse experimental colitis. Lab. Invest. 93:322-333.

Liu, C. J., M. J. Paape, and R. R. Peters. 1993. Flow cytometric leukocyte counts. Comp. Haematol. Int. 3:237-240.

Mehrzad, J., H. Dosogne, E. Meyer, R. Heyneman, and C. Burvenich. 2001. Respiratory burst activity of blood and milk neutrophils in dairy cows during different stages of lactation. J. Dairy Res. 68:399-415.

Okada, S. S., E. M. de Oliveira, T. H. de Araujo, M. R. Rodrigues, R. C. Albuquerque, R. A. Mortara, N. N. Taniwaki, H. I. Nakaya, A. Campa, and A. C. R. Moreno. 2016. Myeloperoxidase in human peripheral blood lymphocytes: Production and subcellular localization. Cell. Immunol. 300:18-25.

Piepers, S., S. De Vliegher, K. Demeyere, B. N. Lambrecht, A. de Kruif, E. Meyer, and G. Opsomer. 2009. Technical note: Flow cytometric identification of bovine milk neutrophils and simultaneous quantification of their viability. J. Dairy Sci. 92:626-631.

Pilling, D., T. Fan, D. Huang, B. Kaul, and R. H. Gomer. 2009. Identification of markers that distinguish monocyte-derived fibrocytes from monocytes, macrophages, and fibroblasts. PLoS One 4: e7475.

Plessers, E., A. Watteyn, H. Wyns, B. Pardon, P. De Backer, and S. Croubels. 2015. Study of the immunomodulatory properties of gamithromycin and dexamethasone in a lipopolysaccharide inflammation model in calves. Res. Vet. Sci. 103:218-223.

Pomeroy, B., A. Sipkab, S. Klaessigb, and Y. Schukken. 2016. Longitudinal characterization of bovine monocyte-derived dendritic cells from mid-gestation into subsequent lactation reveals nadir in phenotypic maturation and macrophage-like cytokine profile in late gestation. J. Reprod. Immunol. 118:1-8.

Sordillo, L. M., and W. Raphael. 2013. Significance of metabolic stress, lipid mobilization, and inflammation on transition cow disorders. Vet. Clin. North Am. Food Anim. Pract. 29:267.

Tay, S. P., S. K. Cheong, N. H. Hamidah, and O. Ainoon. 1998. Flow cytometric analysis of intracellular myeloperoxidase distinguishes lymphocytes, monocytes and granulocytes. Malays. J. Pathol. 20:91-94.

Trevisi, E., M. Amadori, S. Cogrossi, E. Razzuoli, and G. Bertoni. 2012. Metabolic stress and inflammatory response in high-yielding, periparturient dairy cows. Res. Vet. Sci. 93:695-704.

Wauters, J. 2012. Novel biomarkers for the diagnosis of equine infectious joint disease. Chapter 4.1.2 in Flow cytometric detection of cellular myeloperoxidase: A novel tool for equine leukocyte differentiation. PhD Diss. Department of Pharmacology, Biochemistry and Toxicology, Ghent University, Belgium.

Wauters, J., T. Franck, F. Pille, A. Martens, K. Demeyere, S. Sys, D. Serteyn, F. Gasthuys, and E. Meyer. 2011. Flow cytometric detection of myeloperoxidase in horse neutrophils: A novel technique in equine diagnostic research. Vet. Immunol. Immunopathol. 144:417-422.

Zwahlen, R. D., M. Wyderwalther, and D. R. Roth. 1992. Fc receptor expression, concanavalin-A capping, and enzyme content of bovine neonatal neutrophils-A comparative study with adult cattle. J. Leukoc. Biol. 51:264-269. 